\title{
Artificial Intelligence, the Need of the Hour
}

\section{Sarwat Hussain}

How to cite: S Hussain. Artificial intelligence, the Need of the hour.Esculapio 2021;17(01):1-2

DOI: https://doi.org/10.51273/esc21.2517122

\section{Introduction}

$\mathrm{F}$ ourth Industrial revolution is currently sweeping the high-income countries (HIC) with Artificial Intelligence (AI) based automation affecting virtually every aspect of life. The term AI was first coined by McCarthy in 1956. It was not until 2000s that AI began to thrive. The evolution of AI into the current status occurred in the last decade owing to the enhanced computing power using Graphic Processing Units (GPU), development of high-powered computer languages, and the emergence of the Big Data. The latter is generated through wireless communication between 'Smart' sensors/devices and self-learning machines. The word 'smart' is applied to any device that has memory and is able to connect with data networks such as the internet and the processors. In the last few years, there has been exponential growth in AI applications. This can be judged by the projection that the AI field will add \$ 15 Trillion to global economy, by the year 2030, up from \$ 600 Million in 2016. This will occur mostly in the HIC. The adoption of AI by low- and middle-income countries (LMIC) lags far behind that of HICs. The LMICs would miss out in the economic benefits, further widening the global inequalities.

Machine Learning and Deep Learning are branches of AI that are beginning to form the basis of the automation of financial and business decisions, and are the tools of self-driving cars, industrial production, data analytics, quality improvement and healthcare processes to name a few. In healthcare, some of the AI applications have shown to enhance patient care, reduce medical errors, support clinical and administrative decision making, automate equipment maintenance and help reduce operational cost. For instance, AI led cost reductions achieved up to 25

Sarwat Hussain

Professor of Radiology, University of Massachusetts, USA

sarwathussain@hotmail.com percent drop in the length of hospital stay and up to 91 per cent reduction in admissions to step down facilities. In the United States alone, by the year 2026, AI in healthcare is estimated to realize $\$ 150$ billion in annual cost savings.

Artificial Neural Network (ANN), another branch of $\mathrm{AI}$, has made Computer Vision possible. In radiology, pathology, dermatology and ophthalmology, images lend themselves to classification into malignant and benign lesions on mammography and histopathology, confirm presence of diabetic retinopathy and diagnose skin lesions. All this can be achieved by primary care doctors and nurses after some training on the related equipment. In laboratory medicine, AI is employed in precluding unnecessary tests, improving patient safety, and initiating alerts for abnormal results. In advanced laboratories of molecular/genomic testing, AI can be used to accurately identify genetic variants and match up with possible treatments.

Medical Robots, using AI technology, are assisting doctors from minimally invasive procedure to open heart surgery. Humanoid robots work for indigent patients, distribute medication and documents within the hospital premises, and help engineers in equipment maintenance.

In the many low-income countries, $\mathrm{AI}$ is limited to medical imaging and clinical laboratory machines. Computed Tomography, Magnetic Resonance Imaging and mammography machines usually have computer assisted image detection capability, and in laboratory equipment abnormal results alerts. These functions are embedded in the machine software as part of the purchase price.

So far adoption of AI technology in Pakistan has been mostly by default. In the social domain, the ubiquitous 'Siri' of iPhone is one example of AI's application of Natural Language Processing (NLP) and Machine Learning. Of late Pakistan's banking has made signi- 
ficant progress in implementing AI solutions. There are other pockets of AI in Pakistan. As of April 2020, there were 31 AI start-up companies in the country. Of the 174 public and private universities, accredited by Higher Education Commission, only 6 universities and 15 other colleges offer advanced education in AI. In Pakistan, digital data is scant. All paper-based record in the country requires digitization. For the AI research in to be rooted in Pakistan, all future data in the public and the private domains must be collected digitally. Tremendous benefit can be derived from building AI infrastructure, because the nation is rich in young and highly intelligent human resource. Sixty five percent of 220 million Pakistan population is under 30 years of age. Enough of these individuals must be trained to realize full economic and human services potential of AI technology.

A question commonly asked in medical circles, "will AI replace the doctors in the future". The answer would be, doctors who are AI educated will replace in those who are not, especially those physicians seeking to work in the West. Currently the role of AI is entirely assistive and supportive. The complexity of the medical sciences will probably prevent AI from taking over physician's functions completely.
As AI is evolving, it is important to recognize that it is not a silver bullet for the society. In fact, significant issues of transparency, regulatory control, ethics, national security, possible misuse, and built-in biases still need to be worked out.

Notwithstanding the responsibilities of the government of Pakistan and institutions of higher learning, all educated individuals, including doctors, need to develop AI consciousness though self-education. Larger the country wide grass root foot print of the AI movement, the greater the chance of producing world class scholars and researchers for the country.

\section{For further reading:}

1. Mike Moore 05. "What is Industry 4.0? Everything you need to know". TechRadar. November 2019.

2. https://healthitanalytics.com/

3. Paranjape K, Schinkel M, Hammer RD, Schouten $B$ et al. The Value of Artificial Intelligence in Laboratory Medicine: Current Opinions and Barriers to Implementation, American Journal of Clinical Pathology,170: 2020, 1-9 https:// doi. org/ 10.1093/ajcp/aqaa170.

4. (https://tracxn.com/explore/ArtificAIl-Intelligence-Startups-in-Pakistan. 